\title{
BMJ Global Health Governance of the Covid-19 response: a call for more inclusive and transparent decision-making
}

\author{
Dheepa Rajan (D) , ${ }^{1}$ Kira Koch, ${ }^{1}$ Katja Rohrer, ${ }^{1}$ Csongor Bajnoczki, ${ }^{1}$ Anna Socha, ${ }^{2}$
} Maike Voss (D) , ${ }^{3}$ Marjolaine Nicod, ${ }^{2}$ Valery Ridde (D) ${ }^{4}$ Justin Koonin ${ }^{5}$

To cite: Rajan D, Koch $\mathrm{K}$, Rohrer K, et al. Governance of the Covid-19 response: a call for more inclusive and transparent decision-making. BMJ Global Health 2020;5:e002655. doi:10.1136/ bmjgh-2020-002655

Handling editor Seye Abimbola

Received 15 April 2020 Revised 29 April 2020 Accepted 29 April 2020

Check for updates

(C) Author(s) (or their employer(s)) 2020. Re-use permitted under CC BY-NC. No commercial re-use. See rights and permissions. Published by BMJ.

${ }^{1}$ UHC2030 Partnership and Health Systems Governance Collaborative, World Health Organization, Geneva

Switzerland

2UHC2030 Partnership, Geneva, Switzerland

${ }^{3}$ German Institute for International and Security Affairs (SWP), Berlin, Germany ${ }^{4}$ CEPED, Institute for Research on Sustainable Development, IRD-Université de Paris, ERL INSERM SAGESUD, Paris, France ${ }^{5}$ AIDS Council of New South Wales (ACON), Surry Hills, New South Wales, Australia

Correspondence to Dr Dheepa Rajan rajand@who.int

\section{INTRODUCTION}

As SARS-COV-2 (severe acute respiratory syndrome coronavirus 2) ravages the globe, heads of state are making swift decisions to put large swathes of the world's population under mass isolation in the race to heed off Covid-19's lethality, particularly in certain population subgroups. How are these decisions - that affect each and every one of us, some groups disproportionately and regardless of Covid-19 status-made? How far have policy makers and politicians consulted those who have experience and expertise on the secondary effects of lockdowns, social isolation measures and movement restrictions?

We attempted to address these questions with a rapid analysis of 24 countries' Covid-19 task force compositions. The countries were selected to represent a range of geographies and income levels. As far as possible, we focused on governance bodies set up or activated to give scientific, or evidence-based, advice to national decision makers. In some countries, the advisory and decision-making bodies were one and the same, often taking the form of government-only interministerial committees. We excluded committees which were established to focus on a specific area, for example, research related to vaccination; rather, we examined committees whose explicit mandate (based on available information) was to provide advisory guidance on the overall national response.

We scanned publicly available documentation from government websites, media articles, and in specific cases, contacted our networks in governments and health ministries for official documentation. We then researched the task force members' backgrounds and triangulated from different sources to classify them based on their current professional role or area of specialisation. Experts were thus categorised based on the
Summary box

Not all countries make their Covid-19 task force membership list public-the available information varies by country.

- There is currently a predominance of politicians, virologists and epidemiologists in the Covid-19 response at the country level.

- Experts on non-Covid-19 health, social and societal consequences of Covid-19 response measures are for the most part, not included in Covid-19 decisionmaking bodies.

- There is little transparency regarding whom decision-making bodies are consulting as their source of advice and information.

- From the available data on Covid-19 decisionmaking entities, female representation is particularly paltry.

- In addition, civil society is hardly involved in national government decision-making nor its response efforts, barring some exceptions.

We need to be more inclusive and multidisciplinary: the Covid-19 crisis is not simply a health problem but a societal one-it impacts every single person in society one way or another.

- Decision makers need to address more systematically the suffering from mental illness exacerbations, domestic violence, child abuse, child development delays, chronic diseases and so on, during lockdown.

principal reason for their appointment to the task force. For example, a physician with a current public health role would be classified as a public health specialist and not a clinician, the assumption being that their current role is most relevant for the task force. The 'government' or 'Ministry of Health' category was allocated to career civil servants, that is, posts which are usually filled by generalists rather than specialists. Most other task force members, including public health institute staff, were categorised according to their expertise since the rationale for their task force membership is their specific skill set 
(mathematical modeller, virologist, etc) rather than their institutional affiliation.

At least two coauthors independently categorised the task force members and crosschecked categorisations with each other.

\section{HOW INCLUSIVE AND TRANSPARENT IS COVID-19 DECICION- MAKING?}

We highlight a number of key issues, some very worrying, made evident by table 1 :

\section{1.'Evidence' seems to be largely understood to mean research-based evidence, and not necessarily experiential, implementation-based evidence from the field}

The vast majority of Covid-19 response task force members are from reputed universities and government institutes where rigorous research is conducted in the classical sense, often under clinical trial or laboratory conditions. Information and evidence on the lived experiences and everyday challenges faced by the various groups in society who are (at times, severely) affected by isolation measures seem to be altogether overlooked in the urgency of the current situation.

\section{Among researchers, mainly virologists and epidemiologists seem to be consulted, leaving out other health and also non- health experts}

Most countries acknowledge the need for government to work jointly with the medical (and public health) community in the national Covid-19 response. However, mainly virologists and epidemiologists seem to be consulted, largely leaving out specialists in areas such as mental health, child health, chronic diseases, preventive medicine, gerontology, not to mention experts in non-health spheres.

Social isolation measures have enormous secondary effects ${ }^{1}$ beyond the primary aim of curbing viral spread. These effects go far beyond health (discussed below). But even within the health space, the consequences of not accessing, or inadequately accessing, ${ }^{2}$ basic essential services for a wide range of non-Covid-19-related conditions $^{3}$ do not seem to have been sufficiently considered.

\section{When the task force is government-only, more non-health sectors seem to be represented, but at the detriment of non- government expertise}

Still, some countries' Covid-19 task forces are governmentonly. In those cases, there at least seems to be a stronger presence of non-health sectors, although to the detriment of non-government expertise.

In a number of countries, Covid-19 task forces consist of high-level government cadres only, combining the advisory and decision-making elements into one. Medical and epidemiological expertise seems to come from government health institutions, but it is not always clear who is being consulted beyond government.

A multiministry task force at least theoretically brings in concerns from other sectors such as education, ${ }^{4}$ economy, interior, and so on, potentially raising serious issues in terms of, for example, child development ${ }^{5}$ (relevant to decisions on school closures, for example) ${ }^{6}$ loss of livelihoods ${ }^{7}$ (particularly relevant in low-income countries $^{8}$ and those with large social inequalities and no social safety net), and further marginalisation of migrants ${ }^{9}$ and illegal workers (who often have nowhere to isolate to). However, how far those concerns are actually taken into consideration is impossible to discern without more transparency with regard to the content of deliberations and potential consultations with external parties.

\section{Civil society and community groups do not seem to be consulted at all}

In addition to civil society and community groups not being engaged in primary discussions, neither are social workers, child development specialists, human rights lawyers, and many other people whose experiential and vocational expertise are particularly relevant in terms of societal rights, and groups affected by isolation measures. The WHO weekly Covid-19 situation update from 15 April mentions that only $36 \%$ of member states reported having a Covid-19 community engagement plan. ${ }^{10}$ In addition, a majority of the 175 civil society respondents from 56 countries confirmed in a recent rapid survey of the UHC2030 Alliance's Civil Society Engagement Mechanism that most of their Covid-19 response work was, indeed, independent of the government. Results and methodology of the survey can be found here.

Vulnerable groups ${ }^{11}$ such as the disabled, ${ }^{12}$ those with serious mental health conditions, ${ }^{13}$ single mothers, ${ }^{14}$ people in abusive family relationships ${ }^{15}$ and the elderly ${ }^{16}$ bear the burden of the negative consequences of isolation and loneliness, potentially threatening the social fabric of society. Civil society organisations, community groups, social workers, nurse-caregivers and many other groups are at the front lines with this broad cross-section of society clearly affected by the far-reaching effects of mass isolation. Civil society can also raise awareness on existing social inequalities which are usually exacerbated in crisis situations, leaving many to feel that 'self-isolation is a privilege for the rich'. ${ }^{17-20}$ If there is one thing that we should learn from another virus-based crisis (HIV), it is that the population, communities and civil society are an integral part of the crisis solution. ${ }^{21}$

\section{Women are a minority in Covid-19 task forces, and are not represented at all in some}

The Women in Global Health movement has already lamented the abysmally low proportion of women represented in global Covid-19 response efforts. ${ }^{22}$ Besides some notable exceptions, the same low percentages of female experts are seen across the national task forces we rapidly reviewed, with some task forces even being all male. Women's perspectives and expertise clearly seem to be heard less often than male colleagues, even while 
Table 1 Covid-19 task forces set up to advise national governments

\begin{tabular}{|c|c|c|c|}
\hline Country & $\begin{array}{l}\text { Name of task force convened or activated for Covid-19 } \\
\text { response }\end{array}$ & $\begin{array}{l}\text { Composition of task force by member } \\
\text { expertise }\end{array}$ & Gender distribution \\
\hline Argentina $^{28}$ & Expert Committee (El comité de expertos) & $\begin{array}{l}5 \text { Government officials } \\
2 \text { Ministry of Health officials } \\
6 \text { Infectious disease specialists } \\
1 \text { Epidemiologist } \\
1 \text { Public health specialist }\end{array}$ & $12 \mathrm{M} ; 3 \mathrm{~F}$ \\
\hline Belgium $^{29} 30$ & $\begin{array}{l}\text { Scientific Committee Coronavirus (Comité scientifique } \\
\text { Coronavirus) }\end{array}$ & $\begin{array}{l}3 \text { Infectious disease specialists } \\
1 \text { Epidemiologist } \\
1 \text { Laboratory specialist }\end{array}$ & $2 \mathrm{M} ; 3 \mathrm{~F}$ \\
\hline Burkina Faso ${ }^{31}$ & Name unknown & $\begin{array}{l}1 \text { Ministry of Health official } \\
4 \text { Infectious disease specialists } \\
2 \text { Epidemiologists } \\
3 \text { Public health specialists } \\
2 \text { Other medical specialists } \\
1 \text { Communication specialist } \\
1 \text { Private sector } \\
4 \text { Unknown }\end{array}$ & $14 \mathrm{M} ; 5 \mathrm{~F}$ \\
\hline Chad $^{32}$ & $\begin{array}{l}\text { Scientific Committee for Covid-19 } \\
\text { (Comité Scientifique Covid-19) }\end{array}$ & $\begin{array}{l}1 \text { Ministry of Health official } \\
7 \text { Infectious disease specialists } \\
1 \text { Epidemiologist } \\
1 \text { Laboratory specialist } \\
8 \text { Public health specialists } \\
2 \text { Intensive Care specialists } \\
12 \text { Other medical specialists } \\
1 \text { Pharmacist } \\
1 \text { Nutrition specialist } \\
1 \text { Lawyer } \\
1 \text { Socioanthropologist } \\
1 \text { Historian }\end{array}$ & $33 \mathrm{M} ; 4 \mathrm{~F}$ \\
\hline Chile $^{33}$ & $\begin{array}{l}\text { Advisory Board of Ministry of Health for Covid-19 } \\
\text { (Consejo Asesor del MINSAL por Covid-19) }\end{array}$ & $\begin{array}{l}2 \text { Ministry of Health officials } \\
1 \text { Infectious disease specialist } \\
3 \text { Public health specialists } \\
1 \text { Other medical specialist }\end{array}$ & $3 \mathrm{M} ; 4 \mathrm{~F}$ \\
\hline China ${ }^{3435}$ & $\begin{array}{l}\text { Central Leading Group on Responding to the Novel Coronavirus } \\
\text { Disease Outbreak }\end{array}$ & - 9 Government officials & $8 \mathrm{M} ; 1 \mathrm{~F}$ \\
\hline \multirow[t]{2}{*}{ France ${ }^{36-39}$} & $\begin{array}{l}\text { Scientific council Covid-19 } \\
\text { (Conseil scientifique Covid-19) }\end{array}$ & $\begin{array}{l}4 \text { Infectious disease specialists } \\
1 \text { Epidemiologist } \\
1 \text { Mathematical modelling specialist } \\
1 \text { Intensive Care specialist } \\
1 \text { Other medical specialist } \\
1 \text { Anthropologist } \\
1 \text { Sociologist }\end{array}$ & $8 \mathrm{M} ; 2 \mathrm{~F}$ \\
\hline & $\begin{array}{l}\text { Analysis, research and expertise committee } \\
\text { (Comité analyse, recherche et expertise (CARE)) }\end{array}$ & $\begin{array}{l}6 \text { Infectious disease specialists } \\
1 \text { Mathematical modelling specialist } \\
2 \text { Laboratory specialists } \\
2 \text { Other medical specialists } \\
1 \text { Anthropologist }\end{array}$ & $7 \mathrm{M} ; 5 \mathrm{~F}$ \\
\hline Germany $y^{40-42}$ & $\begin{array}{l}\text { Interministerial crisis unit } \\
\text { (Krisenstab) }\end{array}$ & $\begin{array}{l}\text { Government officials from six different } \\
\text { ministries }\end{array}$ & Unknown \\
\hline Guinea $^{4344}$ & $\begin{array}{l}\text { Scientific council on pandemic response to coronavirus disease } \\
\text { (Covid-19) } \\
\text { (Conseil scientifique de riposte contre la pandémie de la maladie à } \\
\text { coronavirus (Covid-19)) }\end{array}$ & $\begin{array}{l}2 \text { Infectious disease specialists } \\
1 \text { Epidemiologist } \\
1 \text { Laboratory specialist } \\
3 \text { Public health specialists } \\
3 \text { Pharmacists } \\
3 \text { Other medical specialists } \\
1 \text { Psychologist } \\
1 \text { Economist } \\
2 \text { Socioanthropologist }\end{array}$ & $14 \mathrm{M} ; 3 \mathrm{~F}$ \\
\hline Haiti ${ }^{45}$ & $\begin{array}{l}\text { Scientific committee to combat coronavirus } \\
\text { (Cellule scientifique pour lutter contre le coronavirus) }\end{array}$ & $\begin{array}{l}1 \text { Ministry of Health official } \\
2 \text { Infectious disease specialists } \\
1 \text { Epidemiologist } \\
1 \text { Laboratory specialist } \\
2 \text { Public health specialists } \\
1 \text { Intensive Care specialist } \\
3 \text { Other medical specialists } \\
1 \text { Mental health specialist } \\
1 \text { Sociologist } \\
1 \text { Civil society }\end{array}$ & $12 \mathrm{M} ; 2 \mathrm{~F}$ \\
\hline Hungary ${ }^{46}$ & $\begin{array}{l}\text { Coronaviral Defence Operational Staff } \\
\text { (Koronavirus-járvány Elleni Védekezésért Felelős Operatív Törzs) }\end{array}$ & $\begin{array}{l}11 \text { Government officials } \\
3 \text { Ministry of Health officials } \\
1 \text { Infectious disease specialist }\end{array}$ & $14 \mathrm{M} ; 1 \mathrm{~F}$ \\
\hline
\end{tabular}




\begin{tabular}{|c|c|c|c|}
\hline & $\begin{array}{l}\text { Scientific Technical Committee } \\
\text { (Comitato Tecnico Scientifico) }\end{array}$ & $\begin{array}{l}4 \text { Ministry of Health officials } \\
2 \text { Infectious disease specialists } \\
1 \text { Public health specialist }\end{array}$ & $7 \mathrm{M} ; 0 \mathrm{~F}$ \\
\hline Kenya ${ }^{5051}$ & National Emergency Response Committee & $\begin{array}{l}17 \text { Government officials } \\
4 \text { Ministry of Health officials }\end{array}$ & $15 \mathrm{M} ; 6 \mathrm{~F}$ \\
\hline Mali 5253 & $\begin{array}{l}\text { Crisis Committee } \\
\text { (Le Comité de crise) }\end{array}$ & $\begin{array}{l}2 \text { Governmental officials } \\
2 \text { Ministry of Health officials } \\
1 \text { Infectious disease specialist } \\
2 \text { Laboratory specialists } \\
4 \text { Public health specialists } \\
1 \text { Other medical specialist }\end{array}$ & $12 \mathrm{M} ; 0 \mathrm{~F}$ \\
\hline & $\begin{array}{l}\text { National task force Covid-1919 (National Disaster Risk Reduction } \\
\text { and Management Council - NDRRMC) }\end{array}$ & - 4 Government officials & $4 \mathrm{M} ; 0 \mathrm{~F}$ \\
\hline \multirow[t]{2}{*}{ Portugal $^{5556}$} & Task force Covid-19 & $\begin{array}{l}13 \text { Infectious disease specialists } \\
10 \text { Epidemiologists } \\
12 \text { Public health specialists } \\
1 \text { Intensive Care specialist } \\
5 \text { Other medical specialists } \\
1 \text { Chemist } \\
2 \text { Communication specialists } \\
25 \text { Unknown }\end{array}$ & $26 \mathrm{M} ; 42 \mathrm{~F}$ \\
\hline & $\begin{array}{l}\text { National Public Health Council } \\
\text { (Conselho Nacional de Saúde Pública) }\end{array}$ & $\begin{array}{l}2 \text { Government officials } \\
2 \text { Ministry of Health Officials } \\
5 \text { Infectious disease specialists } \\
1 \text { Epidemiologist } \\
2 \text { Public health specialists } \\
1 \text { Other medical specialist } \\
1 \text { Pharmacist } \\
2 \text { Lawyers } \\
1 \text { Private sector } \\
2 \text { CSO }\end{array}$ & $14 \mathrm{M} ; 6 \mathrm{~F}$ \\
\hline Singapore ${ }^{57}$ & Multi-Ministry Taskforce on Wuhan Coronavirus & $\begin{array}{l}10 \text { Government officials } \\
1 \text { Ministry of Health official }\end{array}$ & $10 \mathrm{M} ; 1 \mathrm{~F}$ \\
\hline
\end{tabular}

Continued 
Table 1 Continued

\begin{tabular}{|c|c|c|c|}
\hline Country & $\begin{array}{l}\text { Name of task force convened or activated for Covid-19 } \\
\text { response }\end{array}$ & $\begin{array}{l}\text { Composition of task force by member } \\
\text { expertise }\end{array}$ & Gender distribution \\
\hline \multirow[t]{7}{*}{ South Korea ${ }^{5859}$} & Central Disease Control Headquarters (KCDC) & $\begin{array}{l}\text { Led by Jung Eun-Kyeong (Director) } \\
\text { Other members unknown }\end{array}$ & $1 \mathrm{~F}$, unknown \\
\hline & Central Disaster and Safety Countermeasures Headquarters & $\begin{array}{l}\text { Led by the Prime Minister (Chung } \\
\text { Sye-kyun) } \\
\text { Other members unknown }\end{array}$ & $1 \mathrm{M}$, unknown \\
\hline & $\begin{array}{l}\text { Central Incidence Management System for Novel Coronavirus } \\
\text { Infection }\end{array}$ & $\begin{array}{l}\text { Led by Minister of Health and Welfare } \\
\text { (Park Neung-hoo) } \\
\text { Other members unknown }\end{array}$ & $1 \mathrm{M}$, unknown \\
\hline & Central Disaster Management Headquarters & $\begin{array}{l}\text { Led by Ministry of Health and Welfare } \\
\text { (Park Neung-hoo) } \\
\text { Other members unknown }\end{array}$ & $1 \mathrm{M}$, unknown \\
\hline & Government-wide Support Centre & $\begin{array}{l}\text { Led by Minister of Public } \\
\text { Administration and Security } \\
\text { Other members unknown }\end{array}$ & $1 \mathrm{M}$, unknown \\
\hline & $\begin{array}{l}\text { Local Disaster and Safety Countermeasures Headquarters (local } \\
\text { municipal governments nationwide) }\end{array}$ & $\begin{array}{l}\text { Led by the head of the local } \\
\text { government } \\
\text { Other members unknown }\end{array}$ & Unknown \\
\hline & $\begin{array}{l}\text { Local quarantine task force (local municipal governments } \\
\text { nationwide) }\end{array}$ & $\begin{array}{l}\text { Led by the head of the local } \\
\text { government } \\
\text { Other members unknown }\end{array}$ & Unknown \\
\hline Spain $^{60}$ & $\begin{array}{l}\text { Scientific Technical Committee Covid-19 } \\
\text { (Comité Cientifico Técnico Covid-19 19) }\end{array}$ & $\begin{array}{l}3 \text { Infectious disease specialists } \\
3 \text { Epidemiologists }\end{array}$ & $3 \mathrm{M} ; 3 \mathrm{~F}$ \\
\hline Switzerland ${ }^{61}$ & Science Task Force & $\begin{array}{l}6 \text { Infectious disease specialists } \\
2 \text { Epidemiologists } \\
1 \text { Mathematical modelling specialist } \\
1 \text { Laboratory specialist } \\
2 \text { Public health specialists } \\
1 \text { Environmental engineering expert } \\
1 \text { Computer science expert } \\
1 \text { Economist } \\
1 \text { Bioethics expert }\end{array}$ & $12 \mathrm{M} ; 4 \mathrm{~F}$ \\
\hline Thailand $^{62}$ & National committee for controlling the spread of Covid-19 & $\begin{array}{l}26 \text { Government officials } \\
2 \text { Ministry of Health officials }\end{array}$ & $28 \mathrm{M} ; 0 \mathrm{~F}$ \\
\hline Vietnam $^{63}$ & $\begin{array}{l}\text { Committee for Covid-19 Prevention and Control } \\
\text { (Tiểu ban giám sát phòng, chống dịch bệnh Covid-19). }\end{array}$ & $\begin{array}{l}5 \text { Government officials } \\
9 \text { Ministry of Health officials }\end{array}$ & $13 \mathrm{M} ; 1 \mathrm{~F}$ \\
\hline \multirow[t]{3}{*}{ United Kingdom ${ }^{64-66}$} & $\begin{array}{l}\text { New and Emerging Respiratory Virus Threats Advisory Group } \\
\text { (NERVTAG) }\end{array}$ & $\begin{array}{l}9 \text { Infectious diseases specialists } \\
1 \text { Epidemiologists } \\
2 \text { Mathematical modelling specialists } \\
1 \text { Public health specialist } \\
1 \text { Intensive Care specialist } \\
1 \text { Sociologist } \\
1 \text { Psychologist }\end{array}$ & $14 \mathrm{M} ; 2 \mathrm{~F}$ \\
\hline & Advisory Committee on Dangerous Pathogens (ACDP) & $\begin{array}{l}1 \text { Government official } \\
12 \text { Infectious disease specialists } \\
1 \text { Mathematical modelling specialist } \\
1 \text { Public health specialist } \\
1 \text { Other medical specialist }\end{array}$ & $13 \mathrm{M} ; 3 \mathrm{~F}$ \\
\hline & Joint Committee on Vaccination and Immunisation (JCVI) & $\begin{array}{l}19 \text { Infectious disease specialists } \\
1 \text { Other medical specialist } \\
1 \text { Lay member (unknown) }\end{array}$ & $12 \mathrm{M} ; 9 \mathrm{~F}$ \\
\hline $\mathrm{USA}^{6768}$ & White House Coronavirus Task Force & $\begin{array}{l}19 \text { Government officials } \\
1 \text { Ministry of Health official } \\
\\
3 \text { Infectious disease specialists }\end{array}$ & $21 \mathrm{M} ; 2 \mathrm{~F}$ \\
\hline
\end{tabular}

the majority of front-line health staff fighting the crisis is female. ${ }^{23}$

\section{More transparency is needed on who is taking decisions and how}

We took great pains to scan a broad variety of websites, newspapers and government documents in several languages within a short amount of time. Still, information on (1) Who is making far-reaching decisions on an unprecedented global and national crisis? (2) How decision makers are reaching their conclusions (ie, who else are they reaching out to for advice)? (3) Which exact positions advisers had? was not always easy to come by. There are signs that some countries' governments and/or Covid-19 task forces are indeed consulting with outside parties ${ }^{24}$ relevant to the secondary consequences of long-term isolation ${ }^{25}$ but this information is generally not clear and transparent. 
In addition, transparency on selection criteria for the task forces themselves is needed to better understand the weight given to the different aspects of the outbreak.

\section{CONCLUSION}

We acknowledge that the information may not be complete, nor completely up to date, given the extremely fast-paced dynamic of the Covid-19 outbreak as well as response measures. We also recognise that Covid-19 task force compositions are not the sole indication of whose voices are included in decision-making. Through the fairly broad range of (mostly) publicly available information analysed, we attempted to understand which groups the task forces were reaching out to within the scope of a rapid analysis. In general, protocols, reports, minutes of task force meetings and lists of externally consulted parties were simply not easily available. Nevertheless, we feel that the broad conclusions we take based on our rapid (but imperfect) analysis still hold based on the information we were able to access. The table above displays the list of countries and their available task force information.

Governments must recognise the multidimensional effects and needs of society ${ }^{26}$ during this Covid-19 crisis and consult more broadly and across disciplines, within health and beyond health, based on a true multisectoral paradigm. More importantly, more transparency is needed regarding who decision-making bodies are listening to as a basis for their decisions. Now more than ever, the voices of those who are at risk of getting left behind need to be heard. ${ }^{27}$ In the end, we must ensure that we do not do more harm than good with the measures in place to protect our at-risk populations.

Twitter Dheepa Rajan @dheepa_rajan, Kira Koch @KiraJKoch, Maike Voss @maike_voss, Valery Ridde @ValeryRidde and Justin Koonin @JustinKoonin

Acknowledgements The authors thank Esther Njoroge and Eliana Monteforte of the UHC2030 Civil Society Engagement Mechanism Advisory Group for information on some countries' task forces and overall appraisal of the article.

Contributors DR wrote the first draft of the article. DR, KK, KR, CB, AS, MV, VR collected and analysed data. MN and JK revised and edited the article. All authors agreed on the final version.

Funding The authors have not declared a specific grant for this research from any funding agency in the public, commercial or not-for-profit sectors.

Competing interests None declared.

Patient consent for publication Not required.

Provenance and peer review Not commissioned; externally peer reviewed.

Data availability statement № additional data are available.

Open access This is an open access article distributed in accordance with the Creative Commons Attribution Non Commercial (CC BY-NC 4.0) license, which permits others to distribute, remix, adapt, build upon this work non-commercially, and license their derivative works on different terms, provided the original work is properly cited, appropriate credit is given, any changes made indicated, and the use is non-commercial. See: http://creativecommons.org/licenses/by-nc/4.0/.

\section{ORCID iDs}

Dheepa Rajan http://orcid.org/0000-0001-8733-0560

Maike Voss http://orcid.org/0000-0002-7534-6722

Valery Ridde http://orcid.org/0000-0001-9299-8266

\section{REFERENCES}

1 The New Humanitarian. Coronavirus and aid: What we're watching, 9-15 April, 2020. Available: https://www.thenewhumanitarian.org/ news/2020/04/09/coronavirus-humanitarian-aid-response [Accessed 11 Apr 2020].

2 Gossage L. Coronavirus means difficult, life-changing decisions for me and my cancer patients, 2020. Available: https://www. theguardian.com/society/2020/mar/19/cancer-patients-coronavirusoutbreak-difficult-decisions [Accessed 10 Apr 2020].

3 Qin A, Wee S-L. 'No Way Out': In China, Coronavirus Takes Toll on Other Patients, 2020. Available: https://www.nytimes.com/2020/ 03/03/world/asia/china-coronavirus-cancer.html [Accessed $10 \mathrm{Apr}$ 2020].

4 Armitage R, Nellums LB. Considering inequalities in the school closure response to COVID-19. Lancet Glob Health 2020;8:e644.

5 Wang G, Zhang Y, Zhao J, et al. Mitigate the effects of home confinement on children during the COVID-19 outbreak. Lancet 2020;395:945-7.

6 World Food Programme. World food programme gears up to support children left without meals due to COVID-19 school closures, 2020. Available: https://www.wfp.org/news/world-food-programme-gearssupport-children-left-without-meals-due-covid-19-school-closures [Accessed 10 Apr 2020]

7 Ellis-Petersen $\mathrm{H}$, Chaurasia $\mathrm{M}$. India racked by greatest exodus since partition due to coronavirus, 2020. Available: https://www. theguardian.com/world/2020/mar/30/india-wracked-by-greatestexodus-since-partition-due-to-coronavirus [Accessed 7 Apr 2020]

8 Le Monde. En Afrique, « agir au plus vite pour éviter l'hécatombe », 2020. Available: http://lirelactu.fr/source/le-monde/bdcab41c-0ab343ca-8bde-7aeff66511b7 [Accessed 10 Apr 2020].

9 Kluge HHP, Jakab Z, Bartovic J, et al. Refugee and migrant health in the COVID-19 response. The Lancet 2020;395:1237-9.

10 World Health Organization. Weekly update on COVID-19, 8-15 April 2020. Geneva: World Health Organization, 2020.

11 Berger ZD, Evans NG, Phelan AL, et al. Covid-19: control measures must be equitable and inclusive. BMJ 2020;368:m1141.

12 Danjou C. Santé mentale, handicap, psychiatrie: les grands oubliés Du confinement, 2020. Available: https://plus.lesoir.be/291082/ article/2020-03-30/sante-mentale-handicap-psychiatrie-les-grandsoublies-du-confinement [Accessed 5 Apr 2020].

$13 \mathrm{Yao} \mathrm{H}$, Chen J-H, Xu Y-F. Patients with mental health disorders in the COVID-19 epidemic. Lancet Psychiatry 2020;7:e21.

14 Cluver L, Lachman JM, Sherr L, et al. Parenting in a time of COVID-19. The Lancet 2020;395:e64.

15 EURACTIV Network. Domestic violence increases in France during COVID-19 lockdown, 2020. Available: https://www.euractiv.com/ section/politics/news/domestic-violence-increases-in-france-duringcovid-19-lockdown/ [Accessed 8 Apr 2020].

16 Armitage R, Nellums LB. COVID-19 and the consequences of isolating the elderly. Lancet Public Health 2020. doi:10.1016/S24682667(20)30061-X. [Epub ahead of print: 19 Mar 2020].

17 Sur P, Mitra E. Social distancing is a privilege of the middle class. for India's slum dwellers, it will be impossible: CNN, 2020. Available: https://edition.cnn.com/2020/03/30/india/indiacoronavirus-social-distancing-intl-hnk/index.html [Accessed $7 \mathrm{Apr}$ 2020].

18 Breese E. Why self-quarantine from coronavirus is a privilege only for the rich, 2020. Available: https://www.indy100.com/article/ coronavirus-quarantine-self-isolation-outbreak-uk-9372811 [Accessed 7 Apr 2020].

19 Big News Network. Self-isolation a privilege not available to poor, 2020. Available: https://www.bignewsnetwork.com/news/ $264508041 /$ self-isolation-a-privilege-not-available-to-poor [Accessed 4 Apr 2020].

20 Valentino-DeVries J, Lu D, Dance GJX. Location data says it all: staying at home during coronavirus is a luxury, 2020. Available: https://www.nytimes.com/interactive/2020/04/03/us/coronavirusstay-home-rich-poor.html?smid=tw-nytimes\&smtyp=cur [Accessed 5 Apr 2020].

21 Bowtell B. Our HIV lesson: exclude politicians and trust the experts - and the people - to confront coronavirus, 2020. Available: https:// www.smh.com.au/national/our-hiv-lesson-exclude-politiciansand-trust-the-experts-and-the-people-to-confront-coronavirus20200305-p5476a.html [Accessed 4 Apr 2020].

22 Dhatt R, Keeling A, Saraki T. Fighting COVID-19 with one hand tied behind our Backs? 2020. Available: https://www.thinkglobalhealth. org/article/fighting-covid-19-one-hand-tied-behind-our-backs [Accessed 10 Apr 2020].

23 Women in Global Health. Operation 50/50: women's perspectives save lives, 2020. Available: https://www.womeningh.org/operation50-50 [Accessed 6 Apr 2020]. 
24 The state government of North Rhine-Westphalia. Ministerpräsident Armin Laschet beruft „Expertenrat Corona“, 2020. Available: https:// www.land.nrw/de/pressemitteilung/ministerpraesident-arminlaschet-beruft-expertenrat-corona [Accessed 7 Apr 2020].

25 Ministry of Health Consumption and Social Welfare Spain. El Ministerio de Sanidad Y El Consejo General de Psicólogos activan un teléfono de apoyo para La población afectada POR La COVID-19, 2020. Available: https://www.mscbs.gob.es/gabinete/ notasPrensa.do?metodo=detalle\&id=4833 [Accessed 3 Apr 2020]

26 UHC2030. Faced by the COVID-19 crisis, it is crucial that world leaders remember their universal health coverage commitments, 2020. Available: https://www.uhc2030.org/news-events/uhc2030news/faced-by-the-covid-19-crisis-it-is-crucial-that-world-leadersremember-their-universal-health-coverage-commitments-555343/ [Accessed 6 Apr 2020].

27 United Nations Statistics Division. Leaving no one behind. The sustainable development goals report 2016. United Nations, 2016.

28 Clarín. Coronavirus en Argentina: quiénes integran El comité de expertos que asesora a Alberto Fernández, 2020. Available: https:// www.clarin.com/sociedad/coronavirus-argentina-integran-comiteexpertos-asesora-alberto-fernandez_0_D5ZVoVBW1.html [Accessed 7 Apr 2020].

29 Centre de Crise. COVID-19 : une gestion de crise collégiale et complexe, 2020. Available: https://centredecrise.be/fr/news/gestionde-crise/covid-19-une-gestion-de-crise-collegiale-et-complexe [Accessed 6 Apr 2020].

30 Federal public service Belgium. Que font les autorités?: Le service public fédéral (SPF) Santé publique, Sécurité de la Chaîne alimentaire et Environnement, 2020. Available: https://www.infocoronavirus.be/fr/que-font-les-autorites-sanitaires/ [Accessed $7 \mathrm{Apr}$ 2020].

31 Ministry of Health Burkina Faso. Organigramme de Comité de Coordonnateur national de la réponse La pandémie de COVID-19. Ouagadougou: Ministry of Health Burkina Faso, 2020.

32 Republic of Chad. Arrête No. 202/PR/MSP/DG/2020, Portant création Du Comité Scientifique COVID-19: République Du Tchad, 2020.

33 Ministry of Health Chile. Presidente Piñera se reúne Con Consejo Asesor del Minsal POR COVID-19, 2020. Available: https://www. minsal.cl/presidente-pinera-se-reune-con-consejo-asesor-delminsal-por-covid-19/ [Accessed 6 Apr 2020].

34 Xinhuanet. 李克强主持召开中央应对新型 状病毒感染肺炎疫情工 作领导小组会议, 2020. Available: http://www.xinhuanet.com/politics/ 2020-01/26/c_1125504004.htm [Accessed 5 Apr 2020].

35 Cai J. Beijing pins hopes on 'guy with the emperor's sword' to restore order in coronavirus-hit Hubei: South China Morning Post, 2020. Available: https://www.scmp.com/news/china/politics/article/ 3050087/beijing-pins-hopes-guy-emperors-sword-restore-ordercoronavirus [Accessed 8 Apr 2020]

36 Demagny X. Coronavirus : qui sont les onze membres du Conseil scientifique qui conseille le gouvernement: France Inter, 2020. Available: https://www.franceinter.fr/societe/coronavirus-quisont-les-onze-membres-du-conseil-scientifique-qui-conseille-legouvernement [Accessed 4 Apr 2020]

37 Ministry of Solidarity and Health France. COVID-19 : Conseil scientifique COVID-19: France, Ministère des Solidarités et de la Santé, 2020. Available: https://solidarites-sante.gouv.fr/actualites/ presse/dossiers-de-presse/article/covid-19-conseil-scientifiquecovid-19 [Accessed 7 Apr 2020].

38 Publique V. Rapports Publics: Vie Publique, 2020. Available: https:// www.vie-publique.fr/rapports [Accessed 6 Apr 2020]

39 Ministry of Solidarity and Health France. Installation Du comité analyse, Recherche et expertise (care), 2020. Available: https:// solidarites-sante.gouv.fr/actualites/article/installation-du-comiteanalyse-recherche-et-expertise-care [Accessed 6 Apr 2020].

40 Federal Ministry of the Interior for construction and home Germany. Coronavirus: Fragen und Antworten: Bundesministerium des Innern, für Bau und Heimat, 2020. Available: https://www.bmi.bund.de/ SharedDocs/faqs/DE/themen/bevoelkerungsschutz/coronavirus/ coronavirus-faqs.html [Accessed 7 Apr 2020].

41 Press and information office of the federal government Germany. Krisenstab beschließt weitere Maßnahmen: Bundesregierung, Presse- und Informationsamt Der Bundesregierung, 2020. Available: https://www.bundesregierung.de/breg-de/aktuelles/krisenstabcoronavirus-1726492 [Accessed 7 Apr 2020].

42 Federal Ministry of the Interior for construction and home Germany. Gemeinsamer Krisenstab BMI / BMG fällt weitere Beschlüsse: Bundesministerium des Innern, für Bau und Heimat, 2020. Available: https://www.bmi.bund.de/SharedDocs/pressemitteilungen/DE/ 2020/03/coronavirus-weitere-massnahmen.html [Accessed 6 Apr 2020].
43 Republic of Guinea. Decret D/2020/080/PRG/SGG Portant nomination des Membres Du Conseil Scientifique de riposte Contre La Pandemie de la Maladie a coronavirus (Covid-19) République de Guinee, 2020.

44 Bangoura M. Guinée: alpha Condé meuble Le Conseil scientifique de riposte Au COVID-19: Guinéenews, 2020. Available: https://www. guineenews.org/guinee-alpha-conde-meuble-le-conseil-scientifiquede-riposte-au-covid-19/ [Accessed 15 Apr 2020].

45 Geffrard R. Le gouvernement crée une cellule scientifique pour lutter contre Le coronavirus: Le Nouvelliste, 2020. Available: https:// lenouvelliste.com/article/214134/le-gouvernement-cree-une-cellulescientifique-pour-lutter-contre-le-coronavirus [Accessed $7 \mathrm{Apr}$ 2020].

46 Ministry of Interior Hungary. Ülésezik a Koronavírus-fertőzés Elleni Védekezésért Felelős Operatív Törzs, 2020. Available: https://www. kormany.hu/hu/belugyminiszterium/hirek/ulesezik-a-koronavirusfertozes-elleni-vedekezesert-felelos-operativ-torzs [Accessed $3 \mathrm{Apr}$ 2020].

47 Presidency of the Council of Ministers Italian Government. Coronavirus, vertice alla Protezione Civile Con IL Presidente Conte, 2020. Available: http://www.governo.it/it/media/coronavirus-verticealla-protezione-civile-con-il-presidente-conte/14160 [Accessed 6 Apr 2020].

48 RaiNews. Protezione Civile, Borrelli nomina Comitato tecnico scientifico per l'emergenza coronavirus, 2020. Available: http://www. rainews.it/dl/rainews/media/Coronavirus-il-punto-stampa-sullasituazione-il-capo-dipartimento-della-Protezione-civile-AngeloBorrelli-8ba5d59c-b5fb-481a-a9d4-99ba712f0190.html [Accessed 7 Apr 2020].

49 Redazione Start Magazine. Task force tech anti Covid-19, ecco i 74 esperti del governo (un po' troppi?): Redazione Start Magazine, 2020. Available: https://www.startmag.it/innovazione/task-forcetech-anti-covid-19-ecco-i-74-esperti-del-governo-un-po-troppi/ [Accessed 7 Apr 2020].

50 Agutu N. Uhuru forms Taskforce to fight coronavirus, 2020. Available: https://www.the-star.co.ke/news/2020-02-28-uhuruforms-taskforce-to-fight-coronavirus/ [Accessed 15 Apr 2020].

51 The Elephant. Executive order No. 2 of 2020 - national emergency response Committee on coronavirus, 2020. Available: https://www. theelephant.info/documents/executive-order-no-2-of-2020-nationalemergency-response-committee-on-coronavirus/ [Accessed $15 \mathrm{Apr}$ 2020].

52 Mali Rof, No D. 2020-000282/MSAS-SG Du 25 FEV 2020 Portant création Du comité de crise pour La gestion des épidémies de coronavirus et de Crimée Congo: République Du Mali, 2020

53 Mali Rof, No D. 2019-002861/MSAS-SG du 24 Dec 2019 portant nomination des membres du comité scientifique et technique de l'institut national de santé publique (INSP): République du Mali, 2020.

54 Government of the Philippines. Philippines' National Task Force (NTF) COVID19, 2020

55 Directorate-General for Health Portugal. Atualização da Task Force para a operacionalização e a implementação de medidas para prevenção e controlo da infeção por novo Coronavírus - COVID-19 previstas no plano de contingência Direção-Geral da Saúde, 2020. Available: https://www.dgs.pt/a-direccao-geral-da-saude/ comunicados-e-despachos-do-director-geral/despacho-n-0062020de-17032020-pdf.aspx [Accessed 5 Apr 2020].

56 Capelo S. Coronavírus. Quem São os conselheiros dA ministra dA Saúde? 2020. Available: https://www.sabado.pt/portugal/detalhe/ coronavirus-quem-sao-os-conselheiros-da-ministra-da-saude [Accessed 6 Apr 2020].

57 Ministry of Health Singapore. Multi-Ministry Taskforce on Wuhan coronavirus; terms of reference (TORS) and composition, 2020. Available: https://www.moh.gov.sg/docs/librariesprovider5/defaultdocument-library/multi-ministry-taskforce-on-wuhan-coronavirusand-tor-final.pdf [Accessed 7 Apr 2020].

58 Ministry of Health and Welfare South Korea. Quarantine system: South Korea, Ministry of health and welfare, 2020. Available: http:// ncov.mohw.go.kr/en/baroView.do?brdld=11\&brdGubun $=111 \&$ dataGubun=\&ncvContSeq=\&contSeq=\&board_id $=[$ Accessed $7 \mathrm{Apr}$ 2020].

59 Ministry of Health and Welfare South Korea. (1.31) regular briefing of the central incidence management system for novel coronavirus infection, 2020. Available: https://www.mohw.go.kr/eng/nw/ nw0101vw.jsp?PAR_MENU_ID=1007\&MENU_ID=100701\&page $=2 \&$ CONT_SEQ=352672 [Accessed 6 Apr 2020].

60 Ministry of Health Consumption and Social Welfare Spain. Se constituye oficialmente El Comité Científico Técnico COVID-19, 2020. Available: https://www.mscbs.gob.es/gabinete/notasPrensa. do? metodo=detalle\&id=4822 [Accessed 6 Apr 2020]. 
61 Swiss Federal Office of Public Health. Coronavirus : la Confédération met en place un organe consultatif scientifique, 2020. Available: https://www.bag.admin.ch/bag/fr/home/das-bag/aktuell/ medienmitteilungen.msg-id-78626.html [Accessed 7 Apr 2020].

62 Ministry of Public Health Thailand. National Committee for controlling the spread of COVID-19. Thailand: Ministry of public health, 2020.

63 Cộng Hòa Xã Hội Chủ Nghĩa Việtnam. Sô: 295/TTr-BYT. Tò̀ Trình. Về việc ban $H \mathrm{NH}$ danh sách th $\mathrm{NH}$ viên, nhiệm vu V quyền hạn của Tiểu ban giám sát phòng, chống dich bênh COVID-19, 2020.

64 Government of the United Kingdom. Scientific Advisory group for emergencies (SAGE): coronavirus (COVID-19) response. Available: https://www.gov.uk/government/groups/scientific-advisorygroup-for-emergencies-sage-coronavirus-covid-19-response\#role [Accessed 7 Apr 2020].

65 Government of the United Kingdom. Advisory Committee on dangerous pathogens. Available: https://www.gov.uk/government/ groups/advisory-committee-on-dangerous-pathogens\#membership [Accessed 8 Apr 2020].

66 Government of the United Kingdom. Joint Committee on vaccination and immunisation. Available: https://www.gov.uk/government/ groups/joint-committee-on-vaccination-and-immunisation [Accessed 7 Apr 2020].

67 Santucci J. What we know about the white house coronavirus Task force now that Mike Pence is in charge, 2020. Available: https://eu. usatoday.com/story/news/politics/2020/02/27/coronavirus-whatwe-know-mike-pence-and-task-force/4891905002/ [Accessed 8 Apr 2020].

68 White House. Vice president Pence and Secretary Azar add key administration Officials to the coronavirus Task force, 2020. Available: https://www.whitehouse.gov/briefings-statements/vicepresident-pence-secretary-azar-add-key-administration-officialscoronavirus-task-force-2/ [Accessed 8 Apr 2020]. 